PROCEEDINGS OF THE

AMERICAN MATHEMATICAL SOCIETY

Volume 129, Number 4, Pages 1031-1035

S 0002-9939(00)05658-6

Article electronically published on October 10, 2000

\title{
THE DIRICHLET-JORDAN TEST AND MULTIDIMENSIONAL EXTENSIONS
}

\author{
MICHAEL TAYLOR
}

(Communicated by Christopher D. Sogge)

\begin{abstract}
If $\mathcal{F}$ is a foliation of an open set $\Omega \subset \mathbb{R}^{n}$ by smooth $(n-1)$ dimensional surfaces, we define a class of functions $\mathcal{B}(\Omega, \mathcal{F})$, supported in $\Omega$, that are, roughly speaking, smooth along $\mathcal{F}$ and of bounded variation transverse to $\mathcal{F}$. We investigate geometrical conditions on $\mathcal{F}$ that imply results on pointwise Fourier inversion for these functions. We also note similar results for functions on spheres, on compact 2-dimensional manifolds, and on the 3-dimensional torus. These results are multidimensional analogues of the classical Dirichlet-Jordan test of pointwise convergence of Fourier series in one variable.
\end{abstract}

Suppose $f \in L^{1}\left(\mathbb{R}^{n}\right)$, with Fourier transform

$$
\hat{f}(\xi)=(2 \pi)^{-n / 2} \int f(x) e^{-i x \cdot \xi} d x .
$$

We set

$$
S_{R} f(x)=(2 \pi)^{-n / 2} \int_{|\xi| \leq R} \hat{f}(\xi) e^{i x \cdot \xi} d \xi
$$

When $n=1$, the Dirichlet-Jordan test for pointwise convergence of $S_{R} f(x)$ as $R \rightarrow \infty$ states that, if $f$ has bounded variation, then for each $x \in \mathbb{R}$,

$$
\lim _{R \rightarrow \infty} S_{R} f(x)=\frac{1}{2} \lim _{\varepsilon \searrow 0}[f(x+\varepsilon)+f(x-\varepsilon)] .
$$

This can be established as follows. Pick a function $h(t)$, equal to 0 for $t<0,1$ for $0<t \leq 1$, smooth on $(0, \infty)$, and equal to 0 for $t \geq 2$. Set $h(0)=1 / 2$. By Riemann's localization principle there is no loss of generality in assuming $f$ has compact support. If $f$ has bounded variation, its distributional derivative $f^{\prime}=\mu$ is a (signed) measure, and we have

$$
f(x)=\int h(x-y) d \mu(y)+g(x),
$$

with $g \in C_{0}^{\infty}(\mathbb{R})$. If $f(x)$ is adjusted to equal the right side of (3) at each point of discontinuity, then (4) holds for all $x \in \mathbb{R}$. Then we have

$$
S_{R} f(x)=\int S_{R} h(x-y) d \mu(y)+S_{R} g(x) .
$$

Received by the editors April 29, 1999 and, in revised form, June 22, 1999.

1991 Mathematics Subject Classification. Primary 42B08, 35P10.

Key words and phrases. Fourier series, Dirichlet-Jordan test, Gibbs phenomenon.

The author was partially supported by NSF grant DMS-9600065. 
Obviously $S_{R} g(x) \rightarrow g(x)$ for all $x$. The Dirichlet-Jordan result can then be proven using the following two properties of $S_{R} h$ :

$$
S_{R} h(x) \rightarrow h(x), \quad \text { for every } x \in \mathbb{R}
$$

(including $x=0$ ), and, for some $C<\infty$, independent of $x, R$,

$$
\left|S_{R} h(x)\right| \leq C .
$$

To establish (6), one can appeal to the Dini test, or use localization and smoothness for $x \neq 0$, plus a symmetrization argument to cover the case $x=0$. The bound (7) is a consequence of the analysis of the Gibbs phenomenon for $S_{R} h$. From this, the Dirichlet-Jordan result can be deduced via Lebesgue's dominated convergence theorem. Let us state an abstract version of this last segment of the argument.

Let $(Y, \mathfrak{B})$ be a set with sigma algebra, let $\mu$ be a finite signed measure on $\mathfrak{B}$, and let $X$ be a set. Let $h_{R}: X \times Y \rightarrow \mathbb{C}$ be given, for each $R \in(0, \infty)$. Assume that $h_{R}(x, \cdot)$ is $\mathfrak{B}$-measurable, for each $x \in X, R \in(0, \infty)$, that

$$
\left|h_{R}(x, y)\right| \leq C, \quad \forall x \in X, y \in Y, R \in(0, \infty),
$$

and that

$$
\lim _{R \rightarrow \infty} h_{R}(x, y)=h(x, y), \quad \forall x \in X, y \in Y .
$$

Then

$$
\lim _{R \rightarrow \infty} \int_{Y} h_{R}(x, y) d \mu(y)=\int_{Y} h(x, y) d \mu(y), \quad \forall x \in X .
$$

As mentioned, this is simply a consequence of the dominated convergence theorem. The role played by $X$ here is, in essence, trivial, except for the fact that it arises in nontrivial contexts.

Multidimensional analogues of functions for which (6) -(7) hold arise as follows. Let $\Sigma$ be a smooth $(n-1)$-dimensional surface in $\mathbb{R}^{n}$. Let $\mathcal{C}_{1}(\Sigma)$ denote the set of caustic points of order $\geq 1$, in the terminology used in $\S 10$ of [PT]. (This follows Definition 5.2.3 of Dui], in the case where $\Lambda$ is the Lagrangian flow-out of the unit normal bundle of $\Sigma$.) Let $\mathcal{O}_{\Sigma}$ be an open neighborhood of $\mathcal{C}_{1}(\Sigma)$. Let $h(x)$ be a piecewise smooth function, with compact support, with simple jump across $\Sigma$. For $x \in \Sigma$, set $h(x)$ equal to the mean value of its limits from each side. The fact that

$$
S_{R} h(x) \rightarrow h(x), \quad \forall x \in \mathbb{R}^{n} \backslash \mathcal{O}_{\Sigma},
$$

follows from Proposition 26 in $\S 10$ of $[\mathrm{PT}$ (the result for $x \in \Sigma$ holding by the analysis in $\S 11$ ). The fact that, for any compact $K \subset \mathbb{R}^{n} \backslash \mathcal{O}_{\Sigma}$,

$$
\left|S_{R} h(x)\right| \leq C_{K}, \quad \forall R \in(0, \infty), x \in K,
$$

follows from the analysis of the Gibbs phenomenon in $\S 11$ of [PT] (cf. also [CV]). We note that $\mathcal{C}_{1}(\Sigma)$ is empty when $n=2$. Also, when $n=3, \mathcal{C}_{1}(\Sigma)$ is empty if $\Sigma$ is real analytic and not part of a sphere (as noted by $[\mathrm{K}]$ ).

Now suppose we have a foliation of an open set $\Omega \subset \mathbb{R}^{n}$ by such surfaces. More precisely, suppose we have smooth functions $u_{1}, \ldots, u_{n-1}, v$ on $\Omega$, producing a diffeomorphism

$$
\left(u_{1}, \ldots, u_{n-1}, v\right): \Omega \rightarrow Q \subset \mathbb{R}^{n},
$$


where $Q$ is the open cube $(-\pi, \pi) \times \cdots \times(-\pi, \pi)$. We consider the family of surfaces $\Sigma_{c}=\{x \in \Omega: v(x)=c\}$. Assume that $\mathcal{O}$ is an open neighborhood of the union of the sets $\mathcal{C}_{1}\left(\Sigma_{c}\right)$. Fix $\varphi \in C_{0}^{\infty}(\Omega)$. Let $h_{t}: \Omega \rightarrow \mathbb{R}$ be given by

$$
h_{t}(x)= \begin{cases}1 & \text { if } v(x)>t \\ \frac{1}{2} & \text { if } v(x)=t \\ 0 & \text { if } v(x)<t\end{cases}
$$

Let $K$ be any compact set in $\mathbb{R}^{n} \backslash \mathcal{O}$. Then, for each $g \in C^{\infty}(\Omega)$, we have

$$
\left|S_{R}\left(g \varphi h_{t}\right)(x)\right| \leq C_{K}(g), \quad \forall R \in(0, \infty), x \in K, t \in I=(-\pi, \pi) .
$$

Hence, if we set $\Phi(g)(R, x, t)=S_{R}\left(g \varphi h_{t}\right)(x)$, we have

$$
\Phi: C^{\infty}(\Omega) \rightarrow L^{\infty}((0, \infty) \times K \times(-\pi, \pi)) .
$$

Now, if we compose this with the inclusion $\iota: L^{\infty}((0, \infty) \times K \times(-\pi, \pi)) \rightarrow$ $L_{\text {loc }}^{\infty}((0, \infty) \times K \times(-\pi, \pi))$, it is easy to see that the map

$$
\iota \circ \Phi: C^{\infty}(\Omega) \rightarrow L_{\mathrm{loc}}^{\infty}((0, \infty) \times K \times(-\pi, \pi))
$$

is continuous. It follows that the map $\Phi$ in (15) has closed graph. Hence, we can apply the closed graph theorem and deduce that

$$
\sup _{x \in K, t \in I, R \in(0, \infty)}\left|S_{R}\left(g \varphi h_{t}\right)(x)\right| \leq C_{K}\|g\|_{H^{\ell}(\Omega)},
$$

for some finite $\ell$. This estimate can also be demonstrated by a recollection of what makes geometrical optics constructions work, up to any given finite order, and its implementation for the analysis of the Gibbs phenomenon in $\overline{\mathrm{PT}}$. (It would be of interest to study the optimal value of $\ell$, but we will not pursue this here. We will stipulate that $\ell>n / 2$.)

Now, if $\mu$ is a finite (signed) measure on $I$ we can say that, for each $g \in H^{\ell}(\Omega)$,

$$
f(x)=\int_{I} g(x) \varphi(x) h_{t}(x) d \mu(t) \Rightarrow S_{R} f(x) \rightarrow f(x), \quad \forall x \in \mathbb{R}^{n} \backslash \mathcal{O} .
$$

This class of synthesized functions is somewhat constrained, but it will serve as a starting point for an analysis of a much more natural class of functions, which we will now introduce.

Let $\Omega \subset \mathbb{R}^{n}$ be open and let $\mathcal{F}=\left\{\Sigma_{c}: c \in I\right\}$ be a foliation of $\Omega$ by smooth $(n-1)$-dimensional surfaces. Let $\mathcal{M}(\Omega)$ denote the space of finite (signed) Borel measures on $\Omega$. We say

$$
f \in \mathcal{B}(\Omega, \mathcal{F})
$$

if $f$ is a compactly supported element of $L^{\infty}(\Omega)$ with the property that

$$
X_{1} \cdots X_{k} f \in \mathcal{M}(\Omega) \text {, }
$$

for any $k$, and any smooth vector fields $X_{1}, \ldots, X_{k}$ on $\Omega$, provided that at most one of them is not tangent to $\mathcal{F}$. One would have the same class of functions if one insisted the one exceptional vector field be $X_{1}$ (or that it be $X_{k}$ ). The following is our main result.

Theorem 1. Given $f \in \mathcal{B}(\Omega, \mathcal{F})$, there exists a Borel measurable $\tilde{f}$, equal to $f$ a.e., such that, as $R \rightarrow \infty$,

$$
S_{R} f(x) \rightarrow \tilde{f}(x), \quad \forall x \in \mathbb{R}^{n} \backslash \mathcal{O},
$$

where $\mathcal{O}$ is a neighborhood of the union of $\mathcal{C}_{1}\left(\Sigma_{c}\right), c \in I$. 
To begin the proof, we note that $\mathcal{B}(\Omega, \mathcal{F})$ is clearly a module over $C_{0}^{\infty}(\Omega)$. Hence, using a partition of unity, we can assume that $\Omega$ is as in (13), and $\Sigma_{c}=\{v=c\}$. Use the inverse of the diffeomorphism in (13) to pull $f$ back to a compactly supported element $g \in L^{\infty}(Q)$, with the property on $\omega=\partial g / \partial x_{n}$ that

$$
\Delta_{T}^{M} \omega \in \mathcal{M}(Q), \quad M=0,1,2, \ldots
$$

where

$$
\Delta_{T}=\frac{\partial^{2}}{\partial x_{1}^{2}}+\cdots+\frac{\partial^{2}}{\partial x_{n-1}^{2}} .
$$

For the first $n-1$ factors of $(-\pi, \pi)$ in $Q$, throw in the endpoints and identify them, to regard $\omega$ as a compactly supported measure on $\mathbb{T}^{n-1} \times(-\pi, \pi)$. We have, for $\varphi$ continuous on $[-\pi, \pi]$,

$$
\left|\left\langle\varphi(t) e^{-i k \cdot x^{\prime}}, \Delta_{T}^{M} \omega\right\rangle\right| \leq C_{M}\|\varphi\|_{L^{\infty}},
$$

with $x^{\prime}=\left(x_{1}, \ldots, x_{n-1}\right) \in \mathbb{T}^{n-1}, k \in \mathbb{Z}^{n-1}$, so

$$
\left|\left\langle\varphi(t) e^{-i k \cdot x^{\prime}}, \omega\right\rangle\right| \leq C_{M}\langle k\rangle^{-M}\|\varphi\|_{L^{\infty}} .
$$

Hence, we have measures $\mu_{k}$ on $(-\pi, \pi)$, supported on $[-a, a]$ for some $a<\pi$, such that

$$
\left\|\mu_{k}\right\|_{\mathcal{M}(I)} \leq C_{M}\langle k\rangle^{-2 M}, \quad \omega=\sum_{k} e^{i k \cdot x^{\prime}} \mu_{k},
$$

where the norm denotes the total variation of $\mu_{k}$. Hence

$$
g\left(x^{\prime}, y\right)=\int_{-\pi}^{y} \sum_{k} e^{i k \cdot x^{\prime}} d \mu_{k}(t)
$$

so

$$
\begin{aligned}
f(x) & =\sum_{k} e^{i k \cdot u(x)} \int_{-\pi}^{v(x)} d \mu_{k}(t) \\
& =\varphi(x) \sum_{k} e^{i k \cdot u(x)} \int_{-\pi}^{v(x)} d \mu_{k}(t) \\
& =\sum_{k} \varphi(x) g_{k}(x) \int_{-\pi}^{v(x)} d \mu_{k}(t),
\end{aligned}
$$

where we choose $\varphi \in C_{0}^{\infty}(\Omega)$ equal to 1 on the support of $f$, and set $g_{k}(x)=e^{i k \cdot u(x)}$, with $u(x)=\left(u_{1}(x), \ldots, u_{n-1}(x)\right)$. The estimates done above imply convergence in sup-norm of the infinite series, to a function $\tilde{f}(x)$ equal a.e. to $f(x)$. The analysis done above also shows that, for

$$
f_{k}(x)=\varphi(x) g_{k}(x) \int_{-\pi}^{v(x)} d \mu_{k}(t)
$$

we have

$$
S_{R} f_{k}(x) \rightarrow f_{k}(x), \quad x \in \mathbb{R}^{n} \backslash \mathcal{O},
$$

and, for each compact $K \subset \mathbb{R}^{n} \backslash \mathcal{O}$,

$$
\sup _{R \in(0, \infty), x \in K}\left|S_{R} f_{k}(x)\right| \leq C_{K}\left\|g_{k}\right\|_{H^{\ell}(\Omega)}\left\|\mu_{k}\right\|_{\mathcal{M}(I)} .
$$


Now

$$
\left\|g_{k}\right\|_{H^{\ell}(\Omega)} \leq C\langle k\rangle^{\ell}
$$

so, given $N$, we can produce $M=M(\ell, N)$ and apply (25) to obtain

$$
\sup _{R \in(0, \infty), x \in K}\left|S_{R} f_{k}(x)\right| \leq C_{K N}\langle k\rangle^{-N} .
$$

Thus, from (27), we have, for $x \in K$,

$$
\lim _{R \rightarrow \infty} S_{R} f(x)=\sum_{k} f_{k}(x)=\tilde{f}(x),
$$

and the theorem is proven.

It is clear what sort of representative of the class of $f \in \mathcal{B}(\Omega, F)$ the function $\tilde{f}(x)$ is. If $x_{0} \in \Sigma_{c} \subset \Omega$, then $\tilde{f}\left(x_{0}\right)$ is the mean of the limit of $\tilde{f}(x)$ as $x \rightarrow x_{0}$ from within $\{v(x)>c\}$ and as $x \rightarrow x_{0}$ from within $\{v(x)<c\}$. In particular, for each $x_{0} \in \Omega$,

$$
\tilde{f}\left(x_{0}\right)=\lim _{r \searrow 0} \frac{1}{V_{n} r^{n}} \int_{|y|<r} f\left(x_{0}+y\right) d y,
$$

where $V_{n}$ is the volume of the unit ball in $\mathbb{R}^{n}$.

There are other Riemannian manifolds $M$ besides $\mathbb{R}^{n}$ for which there are analogues of Theorem 1, with

$$
S_{R} f(x)=\chi_{R}(\sqrt{-\Delta}) f(x),
$$

where $\Delta$ is the Laplace-Beltrami operator on $M$ and $\chi_{R}(\lambda)$ is 1 for $|\lambda|<R, 0$ for $|\lambda|>R$, and $1 / 2$ for $|\lambda|=R$. One class of examples is the class of "strongly scattering manifolds," in the terminology of $[\mathrm{PT}], \S 10$. Using the "compactification" trick from $\S 6$ of $[\overline{\mathrm{PT}}$, we can extend Theorem 1 to the case where $M$ is a sphere $S^{n}$, or other compact rank-one symmetric space. Using results of [BC], we can extend Theorem 1 to compact 2-dimensional manifolds (and then $\mathcal{O}$ is empty). Using Theorem 5.4 of [T], we can extend Theorem $\square$ to the case $M=\mathbb{T}^{3}$, as long as all the leaves $\Sigma_{c}$ of $\mathcal{F}$ have nonzero Gauss curvature in $\Omega$.

\section{REFERENCES}

[BC] L. Brandolini and L. Colzani, Localization and convergence of eigenfunction expansions, J. Fourier Anal. 5 (1999), 431-447. CMP 2000:12

[CV] L. Colzani and M. Vignati, Gibbs phenomena for multiple Fourier integrals, J. Approximation Theory 80 (1995), 119-131. MR 95k:42021

[Dui] J. J. Duistermaat, Fourier Integral Operators, Birkhäuser, Boston, 1996. MR 96m:58245

[K] J.-P. Kahane, Le phénomène de Pinsky et la géométrie des surfaces, CRAS Paris 321 (1995), 1027-1029. MR 96m:42018

[PT] M. Pinsky and M. Taylor, Pointwise Fourier inversion: a wave equation approach, J. Fourier Anal. 3 (1997), 647-703. MR 99d:42019

[T] M. Taylor, Pointwise Fourier inversion on tori and other compact manifolds, J. Fourier Anal. 5 (1999), 449-463. CMP 2000:12

Department of Mathematics, University of North Carolina, Chapel Hill, North CarOLINA 27599-3902

E-mail address: met@math.unc.edu 